\title{
A High-Q and Small-Mode-Volume Cavity in Microfibers
}

\author{
Jun-long Kou and Hyuck Choo \\ Department of Electrical Engineering, California Institute of Technology, Pasadena, CA 91125, USA \\ ilkou@caltech.edu
}

\begin{abstract}
We propose a novel approach to confine light in a silica microfiber (MF) cavity using a lattice-constant-varying nanohole array. The MF cavity integrated with an optimally designed nanohole array produces Q-factors $\sim 7 \times 10^{5}$ in the near infrared region while its mode volume remains $\sim\left(\lambda_{\mathrm{e}}\right)^{3}$. Our MF cavity of enhanced performance will find potential applications in fiber lasers, nonlinear fiber optics, cavity quantum electrodynamics (QED), and cavity optomechanics. OCIS codes: (060.2310) Fiber optics; (140.3948) Microcavity devices; (140.3945) Microcavities
\end{abstract}

Research activities in microfibers (MFs) have continued to grow in recent years $[1,2]$. Taking advantages of the unique, versatile properties of MFs, different approaches [3-6] have been theoretically proposed or experimentally demonstrated. A Fabry-Perot cavity or a slot resonator shows good light confinement yet suffers from low Q-factors $\left(\sim 10^{1}-10^{2}\right)$ [3]. On the other hand, knot and coil resonators [4-6] provide high Q-factors $\left(\sim 10^{4}-10^{5}\right)$, but their mode volumes are relatively large. Although smaller optical cavities have been implemented in high-Q MF resonators by incorporating phase-shifted Bragg gratings, the resulting Q-factors were low ( 60) [7].

Here, we propose an MF optical cavity capable of achieving both very high-Q and small mode-volume. Results show that the Q-factor reaches $10^{5}-10^{6}$ while the mode volume is kept close to a cube of the eigenwavelength $\left(\lambda_{\mathrm{e}}\right)^{3}$. This approach could prove to be very useful for studies involving cavity quantum electrodynamics (QED) and cavity optomechanics as well as micro-/nano-scale material applications that would require strong light-matter-interactions.

A schematic diagram of the MF cavity is illustrated in Fig. 1(a). The thin MF section in the middle hosts a series of elongated, racetrack-shaped air holes. The lattice constants of the air holes labeled from $N_{1}$ to $N_{12}$ (also from $N_{-1}$ to $N_{-12}$ ) vary from $\Lambda_{1}$ to $\Lambda_{12}$ (also from $\Lambda_{-1}$ to $\Lambda_{-12}$ ). The region between $N_{-12}$ and $N_{12}$ is defined as the "defect region". Outside the defect region, the lattice constant of the air holes is kept constant at $\Lambda_{12}$ to form "host regions". The unit cell is illustrated in the lower right corner of Fig. 1(a). All the parameters regarding the geometry are labeled there.
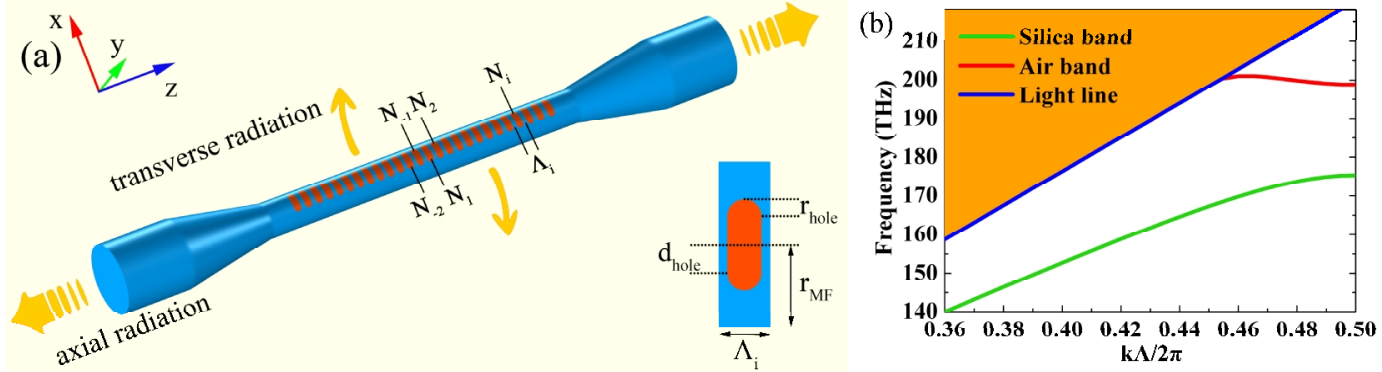

(c)

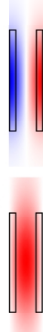

Fig. 1. (a) Schematic diagram of the proposed MF cavity. The air holes (red) are indexed with $N_{\mathrm{i}}$ and the corresponding lattice constant $\Lambda_{\mathrm{i}}$. The cross section of a unit cell in $x-z$ plane $(y=0)$ is shown in the lower right corner. (b) Band structure of a unit cell for $x$-even mode. The band structure is calculated at $r_{\mathrm{MF}}=1 \mu \mathrm{m}, \Lambda=680 \mathrm{~nm}, d_{\text {hole }}=0.5 \Lambda$ and $r_{\text {hole }}=0.3 \Lambda$. Electric field distribution of the (c) silica band mode and (d) air band mode in the $y-z$ plane, respectively.

The introduction of the air hole in a unit cell breaks the symmetry in the $x-y$ plane, resulting in the degeneracy of the Bloch modes. We choose to utilize the $x$-even modes with the electric field mainly polarized in the $x$-direction because these modes exhibit a wider bandgap. The band structure of the host region is shown in Fig. 1(b). A $6.3 \%$ bandgap can be observed from $175.3 \mathrm{THz}$ to $198.8 \mathrm{THz}$. In Fig. 1(c) and (d), we plot the electric field distribution $\left(E_{x}\right)$ at the Brillouin zone boundary. For the mode with a lower eigenfrequency, the electric field mainly resides in the silica region, and we call it "silica band". On the other hand, the field of the mode with a higher eigenfrequency is mostly confined in the air region due to orthogonality. We name this upper band as "air band".

In order to form a cavity, translational symmetry along the $z$-axis must be broken. Here, a parabolic variation of lattice constant (profiles shown in Fig. 2) is introduced in the defect region by gradually decreasing the lattice constants of the air holes from $N_{-12}$ to $N_{-1}$ and $N_{12}$ to $N_{1}$. Thus, the upper bound of the silica band is lifted and resides inside the bandgap of host region. Since the eigenmode of the silica band preserves an odd symmetry with respect to the $x-y$ plane [Fig. 1(c)], an even number of periods is chosen in order to form a first order mode with even symmetry. The first order mode exhibits the strongest electric field or hotspot at the center of the defect. Figure 3(a) displays the detailed electric field distribution of the first order mode in the $x$ direction observed at $\lambda_{\mathrm{e}} \sim 1588 \mathrm{~nm}$. In 
addition, due to the 24 periods present in the defect region, the second order mode also appears, located at $\lambda_{\mathrm{e}} \sim 1643$ $\mathrm{nm}$. Because of the orthogonality requirement, the second order mode forms two hotspot peaks split around the center of the defect that results in more leakage and poorer confinement in the axial direction [Fig. 3(b)]. As a result, the Q-factor of the second order mode $\left(\sim 3.5 \times 10^{3}\right)$ is lower than that of the first order mode $\left(\sim 1.8 \times 10^{4}\right)$.

(a)

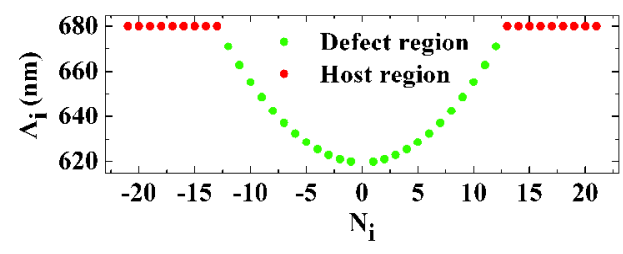

(b)

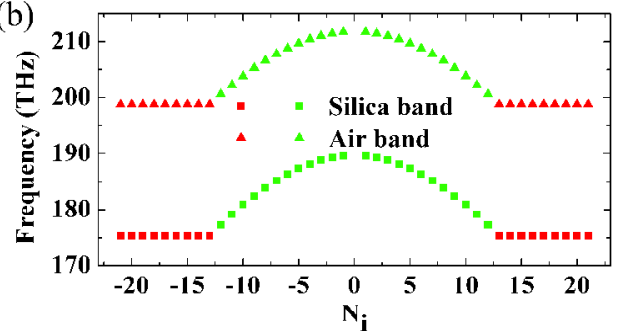

Fig. 2. (a) Lattice constant variation profile and (b) the corresponding band edges of both the silica and air band.

(a) 000000000000000000000000000000000000000 (b) $000000000000 d 0000000000 d 0000000000000000$

Fig. 3. $\mathrm{E}_{\mathrm{x}}$ distribution for (a) the $1^{\text {st }}$ order mode and (b) the $2^{\text {nd }}$ order mode in the $\mathrm{x}-\mathrm{z}$ plane $(\mathrm{y}=0)$.

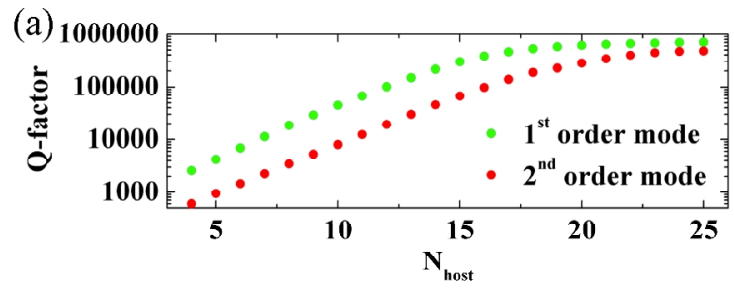

Fig. 4. (a) Q-factor and (b) mode volume versus $\mathrm{N}_{\text {host }}$. The number of holes in the defect region is fixed at 24. (b)

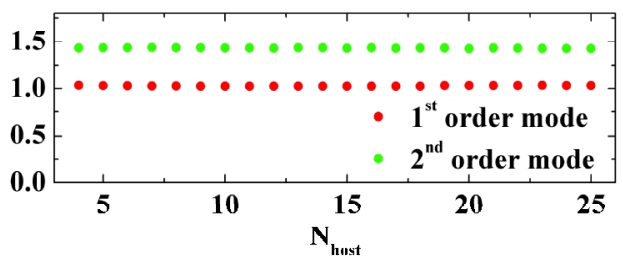

To achieve even higher Q-factor, we increase the length of the host region by adding more periods. It is clearly seen in Fig. 4(a) that as the number of periods in each side of the host regions $\left(N_{\text {host }}\right)$ grows, the Q-factor initially increases almost exponentially because the axial radiation is gradually and effectively suppressed. However, Qfactor ultimately saturates at $\sim 7 \times 10^{5}$ and $\sim 5 \times 10^{5}$ for the first and second order mode when $N_{\text {host }}$ increases beyond 20 and 25, respectively. At these values, the mode is optimally confined in the cavity along the axial direction. Only transverse radiation perpendicular to the optical axis limits further increase in Q-factor. We also calculate the mode volume $\left(V_{\text {eff }}\right)$ of the cavity, shown in Fig. 4(b). Because the mode energy is mostly confined in the defect region with exponentially decaying tail in the host region, $V_{\text {eff }}$ is almost independent of $N_{\text {host }}$, and $V_{\text {eff }}$ stays approximately at $\left(\lambda_{\mathrm{e}}\right)^{3}$ and $1.5\left(\lambda_{\mathrm{e}}\right)^{3}$ for the first and second order mode, respectively.

In summary, we have proposed and analyzed a new MF-based cavity. Its gradually varying lattice constant of the periodic holes along the axial direction minimizes scattering losses during mode-volume compression, and its supported $1^{\text {st }}$ and $2^{\text {nd }}$ modes bearing great resemblance to the locally supported eigenmode are strongly localized in the defect region. With strong local confinement and efficient focusing, the proposed structure accomplishes both high Q-factors and small mode volumes. It will prove to be very useful in micro-/nanophotonic applications.

\section{References}

[1] G. Brambilla, "Optical fibre nanowires and microwires: a review," J. Opt. 12, 043001 (2010).

[2] J.-L. Kou, M. Ding, J. Feng, Y.-Q. Lu, F. Xu, and G. Brambilla, "Microfiber-Based Bragg Gratings for Sensing Applications: A Review," Sensors 12, 8861-8876 (2012).

[3] J.-l. Kou, J. Feng, L. Ye, F. Xu, and Y.-q. Lu, "Miniaturized fiber taper reflective interferometer for high temperature measurement," Opt. Express 18, 14245-14250 (2010).

[4] X. S. Jiang, L. M. Tong, G. Vienne, X. Guo, A. Tsao, Q. Yang, and D. R. Yang, "Demonstration of optical microfiber knot resonators," Appl. Phys. Lett. 88, 223501 (2006).

[5] F. Xu and G. Brambilla, "Demonstration of a refractometric sensor based on optical microfiber coil resonator," Appl. Phys. Lett. 92, 101126 (2008).

[6] H. Yi-Chang, P. Tz-Shiuan, and L. A. Wang, "Millimeter-Sized Microfiber Coil Resonators With Enhanced Quality Factors by Increasing Coil Numbers," IEEE Photon. Technol. Lett. 24, 569-571 (2012).

[7] M. Ding, P. F. Wang, T. Lee, and G. Brambilla, "A microfiber cavity with minimal-volume confinement," Appl. Phys. Lett. 99, 051105 (2011). 